\title{
Short-time Fourier Transform of Free Induction Decays for the Analysis of Serum Using Proton Nuclear Magnetic Resonance
}

\author{
Keiko Hirakawa" ${ }^{1 \uparrow}$, Kaoru Koike ${ }^{2 \uparrow *}$, Yoshimasa Kanawaku ${ }^{3}$, Tsuyoshi Moriyama ${ }^{4}$, \\ Norio Sato ${ }^{2}$, Takao Suzuki ${ }^{2}$, Kenichi Furihata ${ }^{5}$ and Youkichi Ohno ${ }^{1,3}$ \\ ${ }^{1}$ Research Laboratory for Magnetic Resonance, Nippon Medical School, 1-1-5 Sendagi, Bunkyo-ku, Tokyo, 113-8602, JAPAN \\ ${ }^{2}$ Department of Primary Care and Emergency Medicine, Kyoto University, Graduate School of Medicine, 54 Kawaharacho, Syogoin, Sakyou-ku, \\ Kyoto City, Kyoto 606-8507, JAPAN \\ ${ }^{3}$ Department of Legal Medicine, Nippon Medical School, 1-1-5 Sendagi, Bunkyo-ku, Tokyo, 113-8602, JAPAN \\ ${ }^{4}$ Department of Media and Image Technology, Tokyo Polytechnic University, 1583 liyama, Atsugi, Kanagawa, 243-0297, JAPAN \\ ${ }^{5}$ P-One Clinic, Keikokai Medical Corporation, 8-1 Yokamachi, Hachioji, Tokyo, 192-0071, JAPAN \\ "These authors contributed equally to this work.
}

\begin{abstract}
Proton nuclear magnetic resonance (NMR) is useful for the analysis of biological samples such as serum. Free induction decays (FIDs) are NMR signals that follow a radio-frequency pulse applied at the resonance frequency. Short-time Fourier transform (STFT) is a basic method for time-frequency analyses. The purpose of this study was to ascertain whether the STFT of FIDs enables the sensitive detection of changes and differences in serum properties. FIDs were obtained from serum collected from young, healthy, male volunteers $\leq 40$ years of age and seniors $\geq 65$ years of age. Temporal changes in the instantaneous amplitudes for the time-domain analysis, fast Fourier transform for frequency-domain analysis, and STFT were applied to the FIDs. The STFT-based spectrogram represented the complex frequency components that changed dynamically over time, indicating that the spectrogram enabled the visualization of the features of an FID. Furthermore, the results of a partial least-squares discriminant analysis demonstrated that the STFT was superior to the other two methods for discriminating between serum from younger and older subjects. In conclusion, the STFT of FIDs obtained from proton NMR measurements was useful for evaluating similarities and dissimilarities in the FIDs obtained from serum samples.
\end{abstract}

Key words: Short-Time Fourier Transform, serum, modal analysis, Nuclear Magnetic Resonance, spectrogram

\section{INTRODUCTION}

Serum is the liquid portion of blood routinely used as a clinical specimen. Serum consists of low-molecular-weight compounds $^{1-3)}$, macromolecules and their degradation products $^{4,5)}$, inorganic ions ${ }^{6,7)}$, and water. Hydrogen nuclei in serum play important roles through intra- and intermolecular interactions with other nuclei. Most of the hydrogen atoms in serum are associated with water molecules or proteins. Water molecules form hydrogen bonds with nitrogen and hydrogen atoms in macromolecules and low-molecular-weight compounds, stabilizing their molecular conformations ${ }^{8-11)}$. Hydrogen bonding also takes place in the interactions between solutes. Thus, serum has its own dynamic features and assumes a supramolecular structure due to the activity of hydrogen nuclei.

Nuclear magnetic resonance (NMR) is a phenomenon that occurs when the nuclei of certain atoms located in a static magnetic field are exposed to a second, oscillating magnetic field ${ }^{12,13)}$. Free induction decays (FIDs) are impulse responses that result from a radio-frequency pulse applied at the resonance frequency during NMR measurements. For proton (i.e., ${ }^{1} \mathrm{H}$ ) NMR, the FID contains information concerning the dynamic characteristics of hydrogen atoms in the sample. An FID represents a composite signal that is the summation of resonant responses generated by individual hydrogen nuclei.

In structural engineering, vibration analysis is frequently used to extract information regarding the dynamic features

\footnotetext{
*Correspondence to: Kaoru Koike, Department of Primary Care and Emergency Medicine, Kyoto University, Graduate School of Medicine, 54 Kawaharacho, Syogoin, Sakyou-ku, Kyoto City, Kyoto 606-8507, JAPAN

E-mail: kkoike@kuhp.kyoto-u.ac.jp

Accepted January 14, 2019 (received for review October 30, 2018)

Journal of Oleo Science ISSN 1345-8957 print / ISSN 1347-3352 online

http://www.jstage.jst.go.jp/browse/jos/ http://mc.manusriptcentral.com/jjocs
} 
of an object ${ }^{14,15)}$. Free vibration is the natural response of a structure to a particular impulse, whereas resonant vibration is mainly associated with the inertial and elastic properties of the materials within a structure. Modal analysis is now widely used to identify the modes of the vibrations of structures ${ }^{16)}$. As the freely decaying impulse responses observed in vibration analysis can be converted into electrical signals, the modes of the structure can be identified based on both the damping and frequency characteristics of the signals. When composite materials are examined, the signals often include many different frequency components at the same time or many different time components at the same frequency ${ }^{17,18)}$. Time-frequency analysis is an effective way to detect these complexities presented in a vibration signal, and the short-time Fourier transform (STFT) is a basic method used in time-frequency analyses ${ }^{19-22)}$.

Serum is a complex and inhomogeneous fluid that contains an enormous number of hydrogen atoms of differing mobility. Thus, FIDs obtained from serum are complex in nature in terms of both the damping and frequency characteristics. We therefore hypothesized that the STFT of FIDs could enable the sensitive detection of changes and differences in serum properties. Here, we report that the STFT of FIDs is effective for the modal analysis of complex and inhomogeneous fluids such as serum.

\section{EXPERIMENTAL}

\subsection{Collection of serum samples}

Serum samples were collected at the P-One Clinic, Keikokai Medical Corporation, Tokyo, Japan. A total of 128 healthy male Japanese volunteers were enrolled in the study, 53 of whom were between 20 and 40 years old, with the remaining 75 volunteers between 65 and 79 years old. None of the volunteers had a history of systemic disease or abnormal laboratory values in the renal and hepatic function tests. Venous blood samples were collected in vacuum tubes, left at room temperature for $30 \mathrm{~min}$, and centrifuged at $1,000-2,000 \times g$ for $10 \mathrm{~min}$ at $4^{\circ} \mathrm{C}$ in a refrigerated centrifuge. Separated serum was stored at $-80^{\circ} \mathrm{C}$ until further use.

The study protocol was approved by the Institutional Review Board of the P-One Clinic and the ethics committee of the Kyoto University Graduate School and Faculty of Medicine (\#E-1592). The study was conducted in accordance with the ethical principles of the Declaration of Helsinki. Written informed consent was obtained from all subjects.

\subsection{Acquisition of FIDs from the serum samples}

Each serum sample $(100 \mu \mathrm{L})$ was mixed with $500 \mu \mathrm{L}$ of deuterium oxide $\left({ }^{2} \mathrm{H}_{2} \mathrm{O}\right)$ (ISOTEC, Sigma-Aldrich, St. Louis, MO, USA) and pipetted into a 5-mm (outer diameter) NMR tube (Wilmad-LabGlass, Vineland, NJ, USA) for an NMR analysis. Solution-state NMR analyses were performed at a proton resonance frequency of $300 \mathrm{MHz}$ (7.05 Tesla) using an ECX ${ }^{\mathrm{TM}}$ NMR spectrometer(JEOL Ltd., Tokyo, Japan), interfaced with a TH5 probe (auto-tunable type) equipped with Delta ${ }^{\mathrm{TM}}$ NMR processing and control software, version 4.3.2 (JEOL Ltd.). The field was locked to the ${ }^{2} \mathrm{H}$ resonance of the ${ }^{2} \mathrm{H}_{2} \mathrm{O}$ solvent. One-dimensional proton NMR signals were automatically acquired at a probe temperature of $25^{\circ} \mathrm{C}$ using the program supplied by JEOL that supports the macro function in Delta ${ }^{\mathrm{TM}}$. FIDs were acquired using a single pulse with a 2.0 s relaxation delay between repeated pulse sequences. The strong signal arising from the free water was suppressed using DANTE presaturation. Other conditions were as follows: radio-frequency pulse width, $5.44 \mu \mathrm{s}$; acquisition time, $1.468 \mathrm{~s}$; repetition time, $3.468 \mathrm{~s}$; spectral width, 5,580 Hz; number of data points, 8,192; and number of steady-state transients, 400. The FID data were saved in JEOL Delta format (JDF).

\subsection{Processing of FID signals}

FIDs were obtained from all 128 serum samples. FID data in JDF were converted to ASCII format as a two-dimensional matrix (8,192 rows and two columns) using the program supplied by JEOL. FID signals were subsequently processed using LabVIEW ${ }^{\mathrm{TM}} 2015$ (version 15.0.1f1, National Instruments Corporation, Austin, TX, USA). Each row included two columns of data expressed as scalar numbers. The first and second columns indicated the real and imaginary portions of the FID, respectively. For each row, the complex number was calculated as follows: $s=a+j b$, where $a$ and $b$ represent scalar numbers in the real and imaginary columns expressed in the corresponding cells, respectively, and $j$ represents the imaginary unit. The value for $s$ was input as a datum to perform the FFT and STFT of FID signals.

\subsection{Calculation of instantaneous amplitudes for a time- domain analysis}

Instantaneous amplitudes were calculated to analyze the temporal changes in the instantaneous energies of the FIDs. The instantaneous amplitude was defined as the square root of the sum of the squares of the real portion (i.e., $a^{2}$ ) and imaginary portion (i.e., $\left.b^{2}\right)^{23)}$. Temporal changes in the instantaneous amplitude data for each FID were expressed as a $1 \times 8,192$ single row. After combining the total rows into a separate $128 \times 8,192$ matrix, the resulting dataset was used to perform a multivariate data analysis.

\subsection{FFT for frequency-domain analysis}

A FFT was performed to convert the time-domain representation of the FIDs into a frequency-domain representa$\operatorname{tion}^{23)}$. In the case of discrete data, a discrete Fourier 
transform was used to map the discrete time sequences into discrete frequency representations. The following equation was used to calculate the value of the discrete Fourier transform using the FFT algorithm ${ }^{24)}$ :

$$
S_{k}=\sum_{n=0}^{N-1} S_{n} \exp \left(-j \frac{2 \pi n k}{N}\right) .
$$

where $S$ represents the value of the discrete Fourier transform, $N$ represents the number of data points $(8,192)$, and $n$ and $k$ represent indices with values ranging from 0 to $N$ -1 .

In this study, the frequency resolution was $0.681 \mathrm{~Hz}$ $(5,580 \mathrm{~Hz} / 8,192$ data points). No pre-processing of $s$, such as combining window functions, was performed before the FFT. The energy for each frequency of the FID signal was then calculated by dividing the product of $S_{k}$ and its complex conjugate by $N^{24)}$. The discrete power spectrum was then produced. The power spectrum data were expressed as a $1 \times 8,192$ single row. After combining the total rows into a separate $128 \times 8,192$ matrix, the resulting dataset was used to perform a multivariate data analysis.

\subsection{STFT for time-frequency analysis}

An STFT was used for the time-frequency analysis to simultaneously describe the energy density of the FID signals with respect to time and frequency ${ }^{23)}$. For cases in which both time and frequency data were represented by discrete values, the following equation was used to compute the discrete STFT at time $m$ and frequency $f^{25,26)}$ :

$$
\operatorname{STFT}[m, f]=\sum_{i=m d M-\frac{t}{2}}^{m d M+\frac{L}{2}-1} s[i] h[i-m d M] \exp \left(-j \frac{2 \pi f i}{F}\right) .
$$

where $h$ is the window function, $L$ is the window length, $d M$ is the step size required to move the sliding window, $m$ is the index spanning the range 0 to $M-1, M-1$ is the round-down operation after dividing the number of data points by $d M, F$ is the FFT block size of the STFT, and $f$ is the index spanning 0 to $F-1$. The parameters used in this study are as follows: $h$ hanning, $L 128, d M 32, F 512$, and M 257.

A spectrogram was generated as a visual representation of the time-frequency analysis. The data for the STFTbased spectrogram, $P_{S P}[m, f]$, were calculated using the equation below ${ }^{26)}$.

$$
P_{S P}[m, f]=\frac{1}{F}|\operatorname{STFT}[m, f]|^{2} .
$$

Each spectrogram datum in the two-dimensional matrix was read from left to right and row by row, and populated in a single row. In this study, each spectrogram datum was reshaped from a $257 \times 512$ two-dimensional matrix into a 1 $\times 131,584$ single row. After combining the total rows into a separate $128 \times 131,584$ matrix, the resulting dataset was used to perform the multivariate data analysis.

\subsection{Multivariate data analysis}

All data calculated using the three methods described above were imported into Unscrambler X software(version 10.4; Camo Software AS, Oslo, Norway) for a partial least squares discriminant analysis (PLS-DA). All data were transformed using standard normal variates to remove scatter effects, by centering and scaling each individual variable. The Wide Kernel PLS algorithm was used for the factor calculation ${ }^{27-29)}$. Important variables were detected for the separation of sample groups according to the loading weights. Cross-validation was used to statistically evaluate and compare learning algorithms by randomly dividing the data into a software-specified optimal number of segments. To estimate the goodness of fit for future predictions using a defined number of factors, $R^{2}$ and $Q^{2}$ were calculated for the explained-variance plots for calibration and validation data sets, respectively.

\subsection{Experiment using albumin solution}

An additional experiment using albumin solution was performed. The purpose of this experiment was to clarify that STFT was effective especially when the samples were complex and inhomogeneous fluids, by comparing the results from two different kinds of samples, i.e., serum and albumin solution. Albumin from human serum(No. 01310501, FUJIFILM Wako Pure Chemical Corp., Osaka, Japan) was dissolved into distilled water (No. 046-16971, FUJIFILM Wako Pure Chemical Corp., Osaka, Japan) and a stock solution was adjusted to a concentration of $22.0 \%$ (w/ w) of albumin. Next, the following two groups of albumin samples were prepared by diluting the stock solution with distilled water. The dilution rates in the high concentration group $(n=5)$ were $1,1.25,1.67,2.5$, and 5 , and those in the low concentration group $(\mathrm{n}=5)$ were $10,12.5,16.7,25$, and 50 . The FIDs were then obtained from the albumin samples, the instantaneous amplitudes were calculated, the FFT and STFT were conducted, and the PLS-DA performed.

\section{RESULTS}

FIDs were acquired from the serum of 128 healthy male volunteers. Representative examples of the line plots of the real portion of the FID are shown in Fig. 1.

The FIDs in Figs. $1 \mathrm{~A}$ and $1 \mathrm{~B}$ were obtained from the serum of two volunteers aged 26 and 70 -years, respectively. The initial strong signals decayed rapidly, whereas the remaining signals decayed over a longer period of time.

The temporal change in the instantaneous amplitude calculated from the same FID shown in Fig. 1A is depicted in Fig. 2A.

A characteristic feature of the FIDs obtained from human serum is that the initial damping rate is quite high. The power spectrum computed from the same FID as shown in Fig. 1A is demonstrated in Fig. 2B. 
$1 \mathrm{~A}$

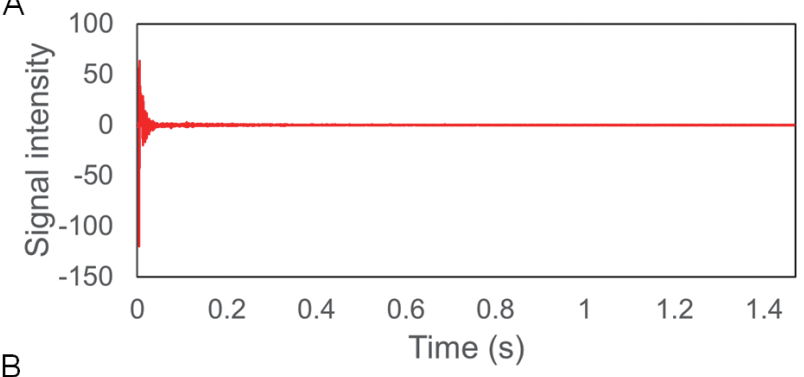

$1 \mathrm{~B}$

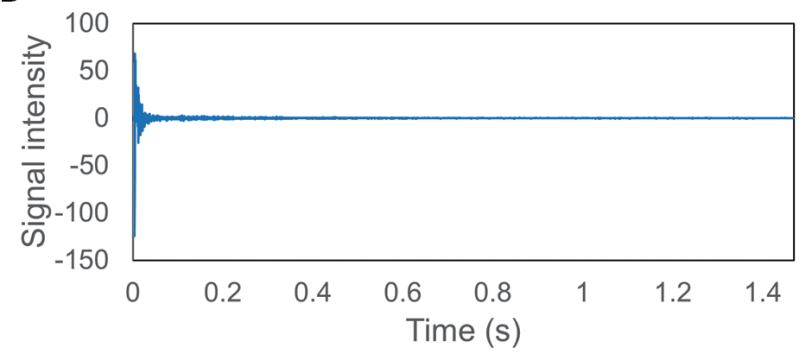

Fig. 1 The FID wave form of proton NMR signals. A) Example of an FID wave form(real portion) obtained from the serum of a 26-year-old healthy male volunteer. B) Example of an FID wave form obtained from the serum of a 70-year-old healthy male volunteer. FID, free induction decay; NMR, nuclear magnetic resonance.

The spectrum is a graph showing the energy content of a signal expressed as a function of frequency. Because serum is a complex and inhomogeneous biological fluid, sharp peaks and broad-band signals originating from different molecules mix and overlap.

The STFT-based spectrogram computed from the same FID, as shown in Fig. 1, is depicted in Fig. 3.

The spectrogram is a graph of the energy content of the signal expressed as a function of frequency and time. The spectrogram shown in Fig. 3 displays time and frequency on the horizontal and vertical axes, respectively; different colors are used to indicate the amplitude of the signal at any given time and frequency, with red and black indicating the highest and lowest energies, respectively. Each row corresponds to the instantaneous power spectrum at a given time. Strong signals were initially present across the frequency axis, but many of them rapidly disappeared. An examination of the remaining signals indicated that those in the broad bands decayed quickly, whereas the others continued for a longer time. A spectrogram such as that presented here enables visualization of the time-frequency feature of FIDs.

A plot of the PLS-DA scores obtained using the instantaneous amplitude data is shown in Fig. 4A, in which each dot represents an individual serum sample.

The results of the calibration versus cross-validation analysis were as follows: $R^{2}=0.910$ and $Q^{2}=0.824$. A plot of the PLS-DA scores obtained using the FFT data $\left(R^{2}=\right.$

\section{$2 \mathrm{~A}$}

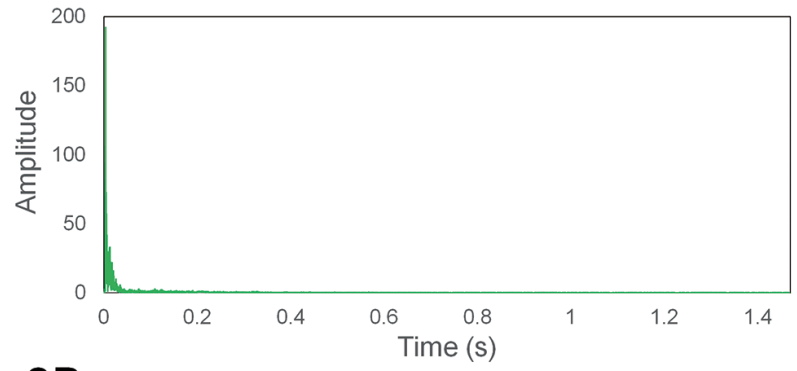

2B

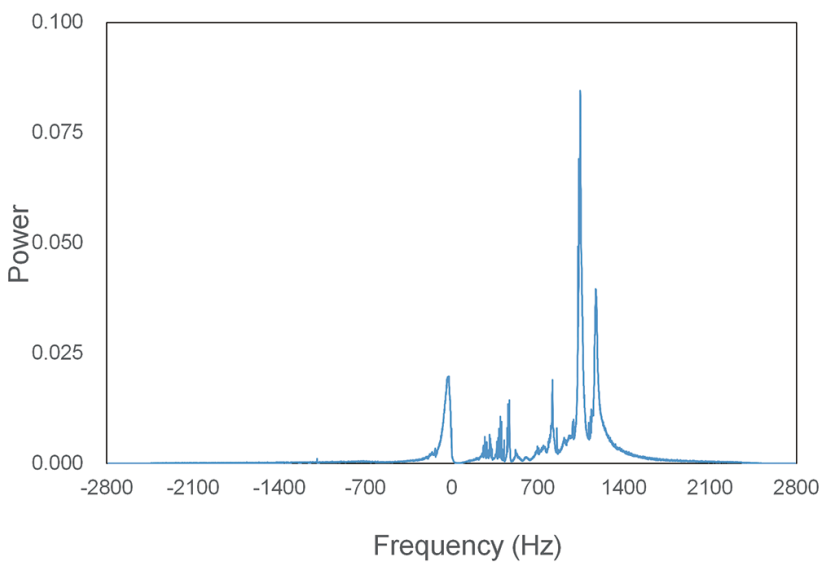

Fig. 2 A) Temporal change in instantaneous amplitude in an FID. The FID used is the same as shown in Fig. 1A. B) Power spectrum calculated by FFT of an FID. The FID used is the same as shown in Fig. 1A. FID, free induction decay; FFT, fast Fourier transform.

0.687 and $\left.Q^{2}=0.649\right)$ is depicted in Fig. 4B, and a plot obtained using the STFT data $\left(R^{2}=0.994, Q^{2}=0.936\right)$ is shown in Fig. 4C. Compared with the other plots, better classifications and clearer separations were observed in the PLS-DA scores plot created from the STFT data.

A plot of the PLS-DA scores obtained using the instantaneous amplitude data is shown in Fig. 5A, in which each dot represents an individual sample of albumin solution.

The results of the calibration versus cross-validation analysis were: $R^{2}=0.904$ and $Q^{2}=0.788$. A plot of PLS-DA scores obtained using the FFT data $\left(R^{2}=0.701\right.$ and $Q^{2}=$ $0.612)$ is depicted in Fig. 5B, and a plot obtained using the $\operatorname{STFT}$ data $\left(R^{2}=0.990, Q^{2}=0.773\right)$ is shown in Fig. 5C. Good classifications and clear separations were observed in the plots of the PLS-DA scores created from the three methods.

\section{DISCUSSION}

Serum is a highly-concentrated, complex, and inhomoge- 


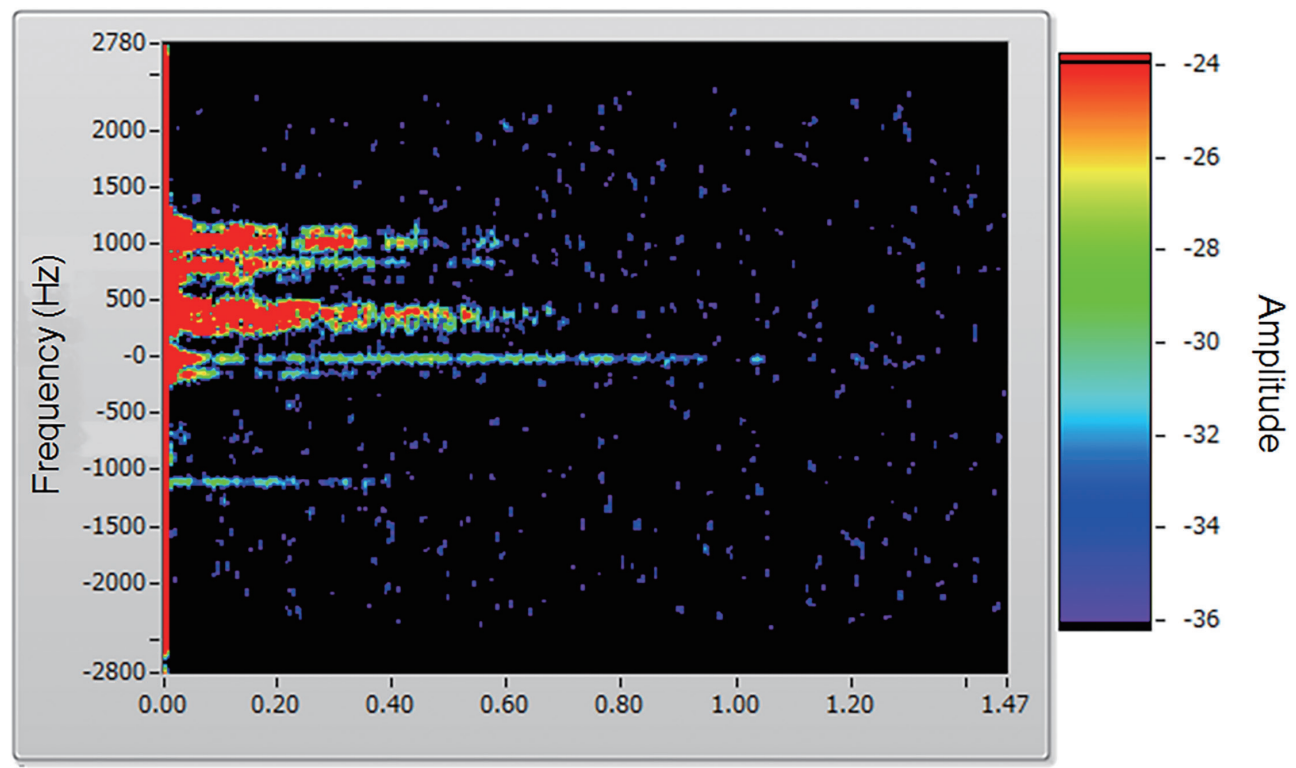

Time (s)

Fig. 3 Spectrogram computed by STFT of an FID. An example of a spectrogram obtained from the same FID shown in Fig. 1A. STFT, short-time Fourier transform; FID, free induction decay.
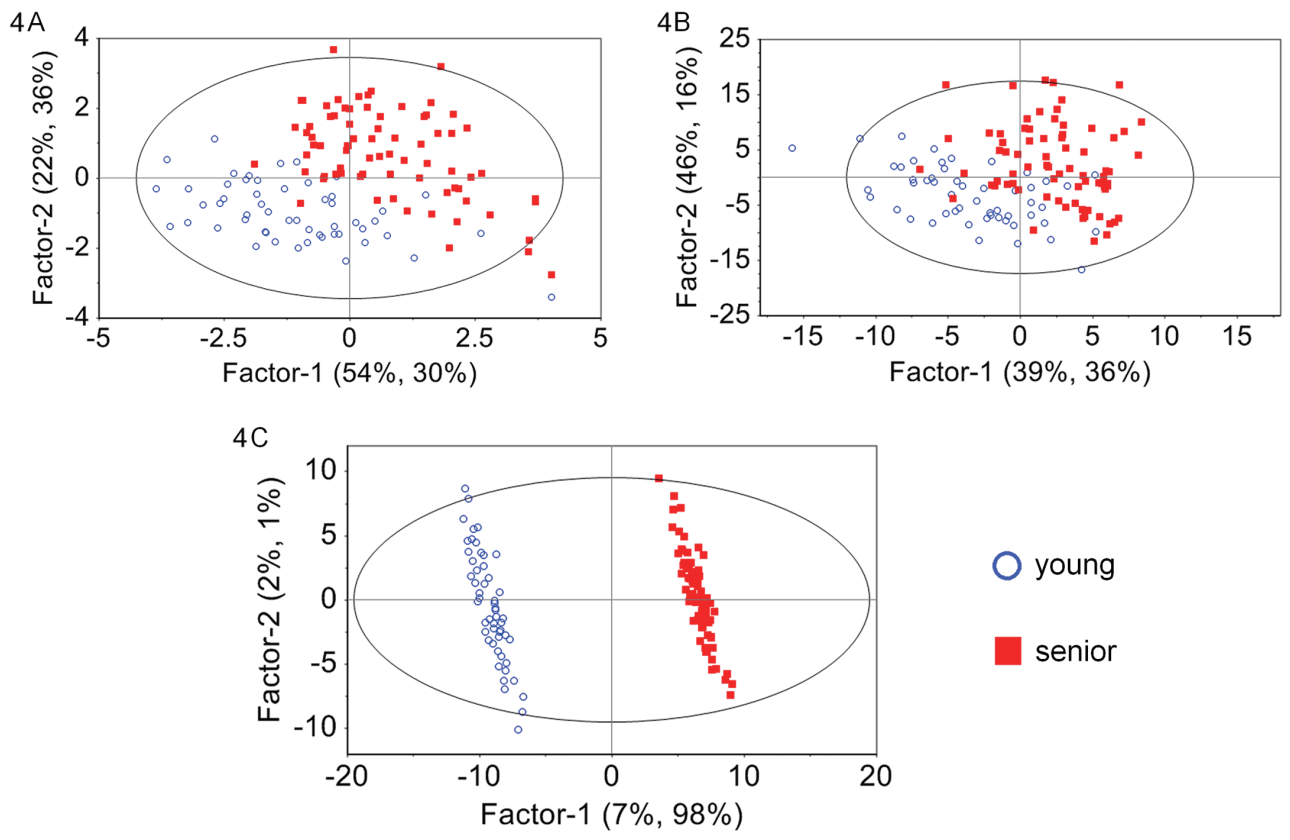

Fig. 4 Plots of PLS-DA scores. FIDs were obtained from serum samples of young $(n=53)$ and senior $(n=75)$ healthy male volunteers. Each point represents an individual sample. A) PLS-DA was performed using FID instantaneous amplitude data $\left(R^{2}=0.910\right.$ and $\left.Q^{2}=0.824\right)$. B) Plot prepared using FID FFT data $\left(R^{2}=0.687\right.$ and $\left.Q^{2}=0.649\right)$. C) Plot prepared using FID STFT data $\left(R^{2}=0.994\right.$ and $\left.Q^{2}=0.936\right)$. Blue open circles, young men; red closed squares, senior men. PLS-DA, partial least squares discriminant analysis; FID, free induction decay; FFT, fast Fourier transform; STFT, short-time Fourier transform.

neous fluid that contains hundreds of different proteins that interact with various other molecules and ions ${ }^{30,31 \text {. }}$. Albumin is the most abundant protein, constituting about $50 \%$ of human serum protein. Albumin is an important carrier of fatty acids, divalent cations, hormones, and bilirubin and plays a role in the maintenance of homeostasis ${ }^{32,33)}$. Lipoproteins interact with cholesterol and triglycerides, and alpha-1-acid glycoproteins interact with basic and neu- 
$5 A$

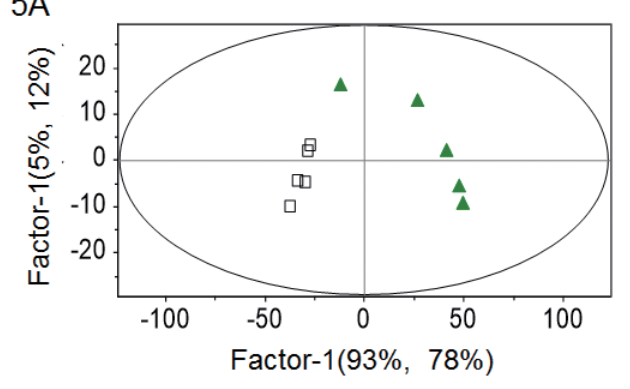

5B

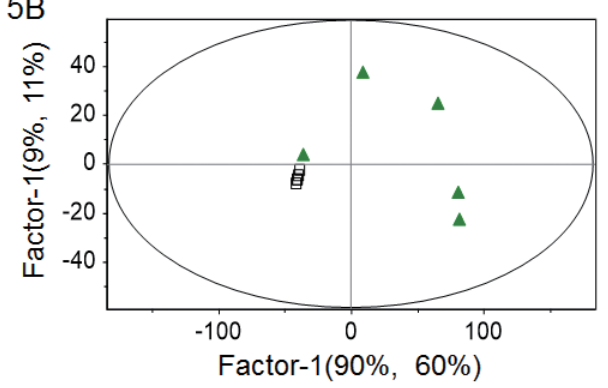

$5 \mathrm{C}$

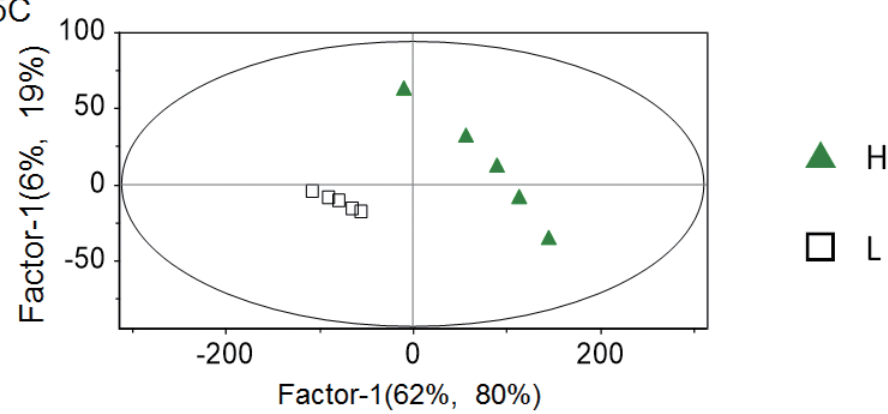

Fig. 5 Plots of PLS-DA scores. FIDs were obtained from two groups of albumin solution samples, i.e., the high and low concentration groups. Each point represents an individual sample. A) PLS-DA was performed using FID instantaneous amplitude data $\left(R^{2}=0.904\right.$ and $\left.Q^{2}=0.788\right)$. B) Plot prepared using FID FFT data $\left(R^{2}=0.710\right.$ and $Q^{2}=$ $0.612)$. C) Plot prepared using FID STFT data $\left(R^{2}=0.990\right.$ and $\left.Q^{2}=0.773\right)$. Green closed triangles, high concentration group $(n=5)$; black open squares, low concentration group $(n=5)$. PLS-DA, partial least squares discriminant analysis; FID, free induction decay; FFT, fast Fourier transform; STFT, short-time Fourier transform.

trally charged lipophilic compounds ${ }^{34)}$. These interactions are reversible and vary with the amounts of individual proteins and corresponding substances. Serum components constantly undergo small conformational changes as a result of interactions between the various molecules ${ }^{35,36)}$. Hydrogen nuclei exist under a diverse array of conditions that are closely related to the supramolecular structure and physical serum properties.

NMR is a powerful, non-invasive analytical tool used in a wide range of applications, including solid-state physics and all branches of chemistry, biology, medical research, and medical diagnosis ${ }^{37-39)}$. Proton NMR encompasses a family of diverse methods and can be divided into two broad categories: time- and frequency-domain analyses.

Time-domain NMR analyses are used in physics to measure spin relaxation time, which can be used to confirm molecular mobility of not only individual components, but also of an entire system with respect to the proton relaxation time of protons ${ }^{40-42}$. For example, in the medical sciences, differences of the proton relaxation time between normal and abnormal tissues can be used in cancer diagnoses ${ }^{43,44)}$. Knowledge gained from studies in these areas provided the basis for the development of magnetic resonance imaging, a technique that is now widely used in medicin ${ }^{45-47)}$. In addition, recent advances in low-field NMR relaxometry have been translated into blood-based diagnostic tests ${ }^{48,49)}$.
Frequency-domain NMR analyses are widely utilized in chemistry and biology in the form of NMR spectroscopy. The information that can be derived from a spectrum (e.g., chemical shift, J coupling, and nuclear Overhauser effect data) is highly useful in identifying chemical structures of molecules ${ }^{39}$. In applications of NMR spectroscopy in medicine, signals originating from specific protons can be selectively used as a means of identifying and quantifying lowmolecular-weight substances, such as metabolites ${ }^{50,51)}$. The profiling of metabolites in serum is now commonly performed in the development of new diagnostic tools for diseases $^{52-54)}$.

In this study, time- and frequency-domain analyses, and STFT were applied to FIDs following the practice used in vibration analysis. We found a report in the literature indicating that the chemical shift dynamics of NMR data can be utilized to determine the intramolecular structure of a specific enzyme and transcription factor ${ }^{55)}$. However, STFT has never been applied to FIDs for evaluating the physicochemical characteristics of a complex and inhomogeneous biological fluid, such as serum.

First, temporal changes in the instantaneous amplitudes of FIDs were examined. The initial strong signals of an FID decayed immediately, and the remaining signals decayed over a longer period of time. The initial signals that rapidly disappeared originated from protons with low mobility, 
whereas the longer-lasting signals were produced by highmobility protons. Low-mobility protons are generally associated with the main chains of macromolecules, whereas high-mobility protons are associated with free low-molecular-weight compounds and macromolecule side chains ${ }^{50,51)}$. A time-domain analysis does not proactively utilize frequency data, and the chemical information of the molecules is not acquired.

The FFT for frequency-domain analysis was then applied to FIDs. In a power spectrum, broad-band signals are derived from low-mobility protons, and peaks are derived from high-mobility protons. The overlapping of signals is caused both by the presence of a variety of molecules and the influence of a complex physicochemical environment ${ }^{51)}$. In the frequency-domain analysis, most of the information was lost with respect to the dynamics of molecules.

Finally, the signal intensity of each frequency component at each time was calculated using STFT. The spectrogram showed that the initial strong signals were spread across the frequency axis. Most of the signals that decayed rapidly originated from low-mobility protons in macromolecules, whereas the signals that decayed over a longer period originated from high-mobility protons. Signals with time-varying frequency content are best represented by a time-frequency distribution. STFT allows the exploitation of features produced by the signal energy concentration in two dimensions (time and frequency), instead of only one (time or frequency). The resulting spectrogram enabled visualization of the FID features using the three dimensions of frequency, time, and signal intensity. Signals originating from individual protons have distinctive damping characteristics associated with their own mobility; thus, the spectrogram represented the complex frequency components that changed dynamically with time.

In proton NMR, the total energy that is released from individual hydrogen nuclei is observed as an FID. Although serum samples are exposed to a static magnetic field during proton NMR measurements, the local magnetic environment around each hydrogen nucleus is heterogeneous due to the presence of other molecules and ions. Consequently, the resonance frequency of each proton signal varies. The mobility of each hydrogen nucleus is affected by a variety of interactions between nuclei, both within the molecules and in neighboring molecules ${ }^{56)}$. The spectrogram reflects the characteristics of protons in serum and provides an overview of the information contained in the FID.

By extracting features from the numerical data generated by each method, we could objectively evaluate similarities and dissimilarities in the FIDs obtained from two groups of serum samples. The PLS-DA score plots indicated that the time- and frequency-domain analyses enabled an effective classification of the groups to a certain extent. However, these two groups were distinctly separated without overlap in the score plot created using the STFT data. In other words, the supramolecular dynamics features of the serum samples were precisely converted to time-frequency characteristics of FIDs. On the other hand, the time-domain analysis, FFT, and STFT were all effective in the case of analyzing a relatively simple and homogeneous mixture, such as the albumin solution. These data indicate that STFT is especially useful when analyzing complex and inhomogeneous fluids.

\section{CONCLUSION}

We demonstrated that STFT is useful for evaluating similarities and dissimilarities in the FIDs obtained from serum samples. The STFT of FIDs could be used to estimate the mode of proton NMR in the analysis of complex and inhomogeneous fluids. This innovative modal analysis method could become a valuable tool for diagnosing a variety of diseases, and assist in identifying applications in other fields of science.

\section{ACKNOWLEDGMENT}

We wish to thank Tomoko Konta and Kaori Okihara, technicians in the Research Laboratory for Magnetic Resonance, Nippon Medical School, for their excellent support in data collection and organization. We gratefully acknowledge the technical advice regarding time frequency analysis provided by Hachiro Imaizumi from Ono Sokki Co., Ltd., Japan. The authors would also like to acknowledge Hideyuki Morikawa and Hideki Muraki from Uniflows Co., Ltd., Japan, who contributed valuable criticisms during the planning of this study.

\section{CONFLICT OF INTEREST}

The authors have declared that no competing interests exist.

\section{AUTHOR CONTRIBUTIONS}

Conceived of and designed the experiments: $\mathrm{KH}$ and $\mathrm{KK}$. Performed the experiments: KH, NS, TS, and KF. Analyzed the data: KH, KK, YK, TM, TS, KF, and YO. Contributed reagents/materials/analysis tools: YK, TM, and NS. Wrote the manuscript: $\mathrm{KH}, \mathrm{KK}$, and $\mathrm{YO}$. 


\section{References}

1) Psychogios, N.; Hau, D.D.; Peng, J.; Guo, A.C.; Mandal, R.; Bouatra, S.; Sinelnikov, I.; Krishnamurthy, R.; Eisner, R.; Gautam, B.; Young, N.; Xia, J.; Knox, C.; Dong, E.; Huang, P.; Hollander, Z.; Pedersen, T.L.; Smith, S.R.; Bamforth, F.; Greiner, R.; McManus, B.; Newman, J.W.; Goodfriend, T.; Wishart, D.S. The human serum metabolome. PLoS ONE 6, e16957 (2011).

2) Dunn, W.B.; Brown, M.; Lin, W.; Broadhurst, D.; Zelena, E.; Vaughan, A.A.; Begley, P.; Halsall, A.; Harding, N.; Knowles, J.D.; Francis-McIntyre, S.; Ellis, D.I.; O’ Hagan, S.; Moseley, C.; Potter, P.; Winder, C.L.; Thornton, P.; McWhirter, C.; Burns, A.; Wu, F.C.W.; Wilson, I.D.; Goodacre, R.; Cruickshank, J.K.; Finn, J.D.; Tseng, A.; Aarons, G.; Benjamin, B.; Chew-Graham, S.; Potts, C.; Zubair, M.; Jayson, G.C.; Pan, M.; Purandare, N.; Haselden, J.N.; Nicholls, A.W.; Kell, D.B. Molecular phenotyping of a UK population: defining the human serum metabolome. Metabolomics 11, 9-26 (2015).

3) Gowda, G.A.N.; Raftery, D. Quantitating metabolites in protein precipitated serum using NMR spectroscopy. Anal. Chem. 86, 5433-5440(2014).

4) Adkins, J.N.; Varnum, S.M.; Auberry, K.J.; Moore, R.J.; Angell, N.H.; Smith, R.D.; Springer, D.L.; Pounds, J.G. Toward a human blood serum proteome. Mol. Cell. Proteomics 1, 947-955(2002).

5) Tirumalai, R.S.; Chan, K.C.; Prieto, D.A.; Issaq, H.J.; Conrads, T.P.; Veenstra, T.D. Characterization of the low molecular weight human serum proteome. Mol. Cell. Proteomics 2, 1096-1103(2003).

6) Iyengar, V.; Wolttlez, J. Trace elements in human clinical specimens: evaluation of literature data to identify reference values. Clin. Chem. 34, 474-481 (1988).

7) Minoia, C.; Sabbioni, E.; Apostoli, P.; Pietra, R.; Pozzoli, L.; Gallorini, M.; Nicolaou, G.; Alessio, L.; Capodaglio, E. Trace element reference values in tissues from inhabitants of the European community I. A study of 46 elements in urine, blood and serum of Italian subjects. Sci. Total Environ. 95, 89-105(1990).

8) Tarek, M.; Tobias, D.J. Role of protein-water hydrogen bond dynamics in the protein dynamical transition. Phys. Rev. Lett. 88, 138101-1-4(2002).

9) Fogarty, A.C.; Laage, D. Water dynamics in protein hydration shells: the molecular origins of the dynamical perturbation. J. Phys. Chem. B 118, 7715-7729 (2014).

10) Baker, E.N.; Hubbard, R.E. Hydrogen bonding in globular proteins. Prog. Biophys. Mol. Biol. 44, 97-179 (1984).

11) Allison, S.D.; Chang, B.; Randolph, T.W.; Carpenter, J.F. Hydrogen bonding between sugar and protein is responsible for inhibition of dehydration-induced protein unfolding. Arch. Biochem. Biophys. 365, 289298(1999).
12) Purcell, E.M.; Torrey, H.C.; Pound, R.V. Resonance absorption by nuclear magnetic moments in a solid. Phys. Rev. 69, 37-38(1946).

13) Bloch, F. Nuclear induction. Phys. Rev. 70, 460-474 (1946).

14) Doebling, S.W.; Farrar, C.R.; Prime, M.B.; Shevitz, D.W. Damage identification and health monitoring of structural and mechanical systems from changes in their vibration characteristics: a literature review. Los Alamos National Laboratory Report LA-13070-MS (1996). [Internet]. https://www.osti.gov/servlets/ purl/249299. Accessed 23 October 2018.

15) Neild, S.A.; Williams, M.S.; McFadden, P.D. Nonlinear vibration characteristics of damaged concrete beams. J. Struct. Engin. 129, 260-268(2003).

16) Yang, Y.; Nagarajaiah, S. Blind modal identification of output-only structures in time-domain based on complexity pursuit. Earthquake Engin. Struct. Dynam. 42, 1885-1905 (2013).

17) Lakhdar, M.; Mohammed, D.; Boudjemâa, L.; Rabiâ, A.; Bachir, M. Damages detection in a composite structure by vibration analysis. Energy Procedia 36, 888-897 (2013).

18) Patel, H.; Jamadar, N.I. Modal analysis of composite structure. Int. J. Adv. Engin. Technol. 3, 71-82 (2016).

19) Li, Z.; Crocker, M.J. Modal analysis using Time-frequency transform. [Internet]. https://pdfs.semanticscholar.org/65a6/1a362922b3a7e5744a3d1d1a777781d 06de2.pdf. Accessed 23 October 2018.

20) Nagarajaiah, S.; Varadarajan, N. Short time Fourier transform algorithm for wind response control of buildings with variable stiffness TMD. Engin. Struct. 27, 431-441 (2005).

21) Nagarajaiah, S.; Basu, B. Output only modal identification and structural damage detection using time frequency \& wavelet techniques. Earthquake Engin. Engin. Vib. 8, 583-605(2009).

22) Nagarajaiah, S.; Yang, Y. Blind modal identification of output-only non-proportionally-damped structures by time-frequency complex independent component analysis. Smart Struct. Syst. 15, 81-97(2015).

23) Cohen, L. Time-frequency analysis. $1^{\text {st }}$ ed. PrenticeHall Inc., New Jersey (1995).

24) National Instruments Corp. Using fast Fourier transforms and power spectra in LabVIEW. [Internet]. http://www.ni.com/white-paper/45/en/. Accessed 23 October 2018.

25) National Instruments Corp. TFA STFT VI. [Internet]. http://zone.ni.com/reference/en-XX/help/371419D-01/ lvtimefreqtk/tfa_stft_spectrogram/. Accessed 23 October 2018.

26) National Instruments Corp. STFT Spectrograms VI. [Internet]. http://zone.ni.com/reference/en-XX/ 
help/371361P-01/lvanls/stft_spectrogram_core/. Accessed 23 October 2018.

27) Lindgren, F.; Geladi, P.; Wold, S. The kernel algorithm for PLS. J. Chemom. 7, 45-59 (1993).

28) Rännar, S.; Lindgren, F.; Geladi, P.; Wold, S. A PLS kernel algorithm for data sets with many variables and fewer objects. Part 1: theory and algorithm. J. Chemom. 8, 111-125(1994).

29) Rännar, S.; Geladi, P.; Lindgren, F.; Wold, S. A PLS kernel algorithm for data sets with many variables and few objects. Part II: cross-validation, missing data and examples. J. Chemom. 9, 459-470 (1995).

30) Pieper, R.; Gatlin, C.L.; Makusky, A.J.; Russo, P.S.; Schatz, C.R.; Miller, S.S.; Su, Q.; McGrath, A.M.; Estock, M.A.; Parmar, P.P.; Zhao, M.; Huang, S.T.; Zhou, J.; Wang, F.; Esquer-Blasco, R.; Anderson, N.L.; Taylor, J.; Steiner, S. The human serum proteome: display of nearly 3700 chromatographically separated protein spots on two-dimensional electrophoresis gels and identification of 325 distinct proteins. Proteomics 3, 1345-1364 (2003).

31) Hu, S.; Loo, J.A.; Wong, D.T. Human body fluid proteome analysis. Proteomics 6, 6326-6353 (2006).

32) Curry, S. Lessons from the crystallographic analysis of small molecule binding to human serum albumin. Drug Metab. Pharmacokinet. 24, 342-357(2009).

33) Fujiwara, S.; Amisaki, T. Fatty acid binding to serum albumin: molecular simulation approaches. Biochim. Biophys. Acta 1830, 5427-5434(2013).

34) Yesylevskyy, S.O.; Ramseyer, C.; Savenko, M.; Mura, S.; Couvreur, P. Low-density lipoproteins and human serum albumin as carriers of squalenoylated drugs: insights from molecular simulations. Mol. Pharm. 15, 585-591 (2018).

35) Feroz, S.R.; Mohamad, S.B.; Lee, G.S.; Malek, S.N.; Tayyab, S. Supramolecular interaction of 6-shogaol, a therapeutic agent of Zingiber officinale with human serum albumin as elucidated by spectroscopic, calorimetric and molecular docking methods. Phytomedicine 22, 621-630 (2015).

36) Barreca, D.; Lagana, G.; Toscano, G.; Calandra, P.; Kiselev, M.A.; Lombardo, D.; Bellocco, E. The interaction and binding of flavonoids to human serum albumin modify its conformation, stability and resistance against aggregation and oxidative injuries. Biochim. Biophys. Acta 1861, 3531-3539(2017).

37) Ernst, R.R.; Bodenhausen, G.; Wokaun, A. Principles of nuclear magnetic resonance in one and two dimensions. $1^{\text {st }}$ ed. Clarendon Press Oxford (1987).

38) Sobol, W.T. Medical applications of NMR. Bull. Magn. Reson. 11, 69-85 (1989).

39) Derome, A.E. Modern NMR techniques for chemistry research. United States: Pergamon Books Inc. (1987).
40) Hahn, E.L. Spin echoes. Phys. Rev. 80, 580-594 (1950).

41) Kuentz, M.; Rothenhäusler, B.; Röthlisberger, D. Time domain ${ }^{1} \mathrm{H}$ NMR as a new method to monitor softening of gelatin and HPMC capsule shells. Drug Dev. Indust. Pharm. 32, 1165-1173(2006).

42) Gombia, M.; Bortolotti, V.; Brown, R.J.S.; Camaiti, M.; Cavallero, L.; Fantazzini, P. Water vapor absorption in porous media polluted by calcium nitrate studied by time domain nuclear magnetic resonance. J. Phys. Chem. B 113, 10580-10586 (2009).

43) Damadian, R.; Zaner, K.; Hor, D.; Maio, T. Human tumors detected by nuclear magnetic resonance. Proc. Natl. Acad. Sci. USA 71, 1471-1473(1974).

44） Kiricuta Jr., I.C.; Simplăceanu, V. Tissue water content and nuclear magnetic resonance in normal and tumor tissues. Cancer Res. 35, 1164-1167 (1975).

45) Liang, Z.P.; Lauterbur, P.C. Principles of magnetic resonance imaging: a signal processing perspective: Spie Optical Engineering Press (2000).

46) Nacher, P.J. Magnetic resonance imaging: from spin physics to medical diagnosis. in The Spin. pp. 159-193 (2007).

47) Grover, V.P.B.; Tognarelli, J.M.; Crossey, M.M.E.; Cox, I.J.; Taylor-Robinson, S.D.; McPhail, M.J.W. Magnetic resonance imaging: principles and techniques: lessons for clinicians. J. Clin. Exp. Hepatol. 5, 246-255 (2015).

48) Cistola, D.P.; Robinson, M.D. Compact NMR relaxometry of human blood and blood components. Trends Analyt. Chem. 83, 53-64(2016).

49) Luo, Z.X.; Fox, L.; Cummings, M.; Lowery, T.J.; Daviso, E. New frontiers in in vitro medical diagnostics by low field $\mathrm{T}_{2}$ magnetic resonance relaxometry. Trends Analyt. Chem. 83, 94-102 (2016).

50) Daykin, C.A.; Foxall, P.J.D.; Connor, S.C.; Lindon, J.C.; Nicholson, J.K. The comparison of plasma deproteinization methods for the detection of low-molecularweight metabolites by ${ }^{1} \mathrm{H}$ nuclear magnetic resonance spectroscopy. Anal. Biochem. 304, 220-230 (2002).

51) Stolzenburg, S.; Toft, H.; Lauridsen, M.B.; Zalloua, P.A.; Baunsgaard, D. Improved quality of ${ }^{1} \mathrm{H}$ NMR spectroscopic data for enhanced metabolic profiling of low molecular weight metabolites in human serum. Metabolomics 7, 270-277(2011).

52) Hao, D.; Sarfaraz, M.O.; Farshidfar, F.; Bebb, D.G.; Lee, C.Y.; Card, C.M.; David, M.; Weljie, A.M. Temporal characterization of serum metabolite signatures in lung cancer patients undergoing treatment. Metabolomics 12, 58(2016).

53) Kromke, M.; Palomino-Schätzlein, M.; Mayer, H.; Pfeffer, S.; Pineda-Lucena, A.; Luy, B.; Hausberg, M.; Muhle-Goll, C. Profiling human blood serum metabolites by nuclear magnetic resonance spectroscopy: a compre- 


\section{K. Hirakawa, K. Koike, Y. Kanawaku et al.}

hensive tool for the evaluation of hemodialysis efficiency. Transl. Res. 171, 71-82 e71-79 (2016).

54) Srivastava, N.K.; Annarao, S.; Sinha, N. Metabolic status of patients with muscular dystrophy in early phase of the disease: in vitro, high-resolution NMR spectroscopy based metabolomics analysis of serum. Life Sci. 151, 122-129(2016).

55) Langmead, C.J.; Donald, B.R. Extracting structural in- formation using time-frequency analysis of protein NMR data. in Proceedings of the Fifth Annual International Conference on Computational biology (ACM). pp. 164-175(2001).

56) Mittermaier, A.; Kay, L.E. New tools provide new insights in NMR studies of protein dynamics. Science 312, 224-228(2006). 